\title{
The effect of concentrated growth factors in the treatment of periodontal intrabony defects
}

\begin{abstract}
Aim: To investigate the effect of concentrated growth factors (CGFs) in human intrabony defect treatment. Methods: Thirty-one intrabony defects were randomly treated with CGFs + bovine porous bone mineral (BPBM) or BPBM alone. Probing depth, clinical attachment level and hard tissue fill were evaluated at baseline and 1 year post surgery. Results: No differences in any of the investigated parameters were observed at baseline. At 1 year post therapy, both groups showed significant improvement in clinical parameters $(p<0.001)$. CGFs + BPBM was more effective than BPBM alone at decreasing probing depth $(4.2 \pm 1.3 \mathrm{~mm}$ vs $3.0 \pm 1.6 \mathrm{~mm})$ and clinical attachment level gain $(3.7 \pm 1.3 \mathrm{~mm}$ vs $2.4 \pm 1.1 \mathrm{~mm} ; \mathrm{p} \leq 0.05)$. A favorable increase of hard tissue fill was noted in CGFs + BPBM group compared with BPBM group $(p>0.05)$. The contents of growth factors in CGFs were statistically higher than those in platelet poor plasma $(p<0.001)$. Conclusion: Addition of CGFs significantly improved clinical effectiveness of BPBM for intrabony defect treatment.
\end{abstract}

Lay abstract: Concentrated growth factors (CGFs) are a new generation of platelets concentrates, which contain abundant growth factors. We assumed that CGFs might promote periodontal (gum) regeneration. In the present study, we used CGFs in the treatment of periodontal intrabony defects. The results at 1 year post surgery indicated that the addition of CGFs significantly improved the clinical effectiveness of bone graft alone. More research is needed to better understand this effect.

First draft submitted: 2 February 2016; Accepted for publication: 13 May 2016; Published online: 15 September 2016

Keywords: bovine porous bone mineral $\bullet$ concentrated growth factors $\bullet$ periodontal intrabony defects $\bullet$ periodontal regeneration

Periodontitis is an inflammatory disease that leads to the loss of tooth-supporting tissues. Tissue loss caused by periodontal disease is typically treated by a variety of regenerative treatment modalities, including bone grafts, guided tissue regeneration (GTR) and growth factors, to reform the tooth's supporting tissues [1-5].

However, a common problem of GTR therapy is the high variability and low predictability of healing outcomes, even as complete and predictable reconstruction of periodontal tissues is difficult to obtain with any therapeutic plan [6,7]. Specifically, the degree of healing depends on both the local characteristics of the intrabony defects and the regenerative potential of the residual periodontal tissues $[4,8,9]$.

Polypeptide growth factors are one of the fundamental elements in tissue engineering, which have shown an important role in the growth and differentiation of cells involved in periodontal wound healing $[10,11]$. Recombinant forms of growth factors may have positive effects in experimental studies but are not practical for clinical application owing to complexity of application and their high costs.
Jing Qiao', Jinyu Duan', Yong Zhang ${ }^{1}$, Yi Chu ${ }^{1}$ \& Changzhou Sun*,1 'Department of Periodontology, Outpatient Center, Peking University School \& Hospital of Stomatology, Beijing, China

*Author for correspondence:

Tel.: +861058595052

Fax: +861062174226

Changzhousun66@126.com 
Platelets, a major resource of autogenous growth factors, are among the first cells to reach a wound site and initiate the healing process [12].

Platelet-rich plasma (PRP) was the first generation of platelet gel used in periodontal regeneration therapy [13-18]. While the potential benefits of this procedure have been critiqued, many of the discrepancies are likely more related to the lack of suitable methodological standardization and definition of the different PRP preparations than to any functional inadequacies, as the protocols and biological and surgical techniques used in the administration of the PRPs differ widely between study groups $[19,20]$.

Platelet-rich fibrin (PRF), the second generation of platelet concentrate products, exhibits the same properties as PRP with the advantages of osteogenicity, a simple preparation process and a lack of bovine thrombin and anticoagulants, as it is produced from autologous blood [21-24].

Concentrated growth factors (CGFs) were first developed by Corigliano in 2010 [25]. CGFs are produced by centrifuging blood samples at alternating and controlled speeds using a special centrifuge (Medifuge, Silfradentsrl, Italy). Different centrifugation speeds permit the isolation of a much larger and denser fibrin matrix richer in growth factors than typically found in PRP or PRF. Rodella et al. observed a fibrin network constituted by thin and thick fibrillar elements, and multiple platelet cell elements were observed forming a cell aggregate trapped among the fibrin network [26]. Their study demonstrated the presence of TGF- $\beta 1$ and VEGF in CGF and red blood cell (RBC) layers, suggesting that an improved CGF isolation procedure could optimize the amount of growth factors in the CGF layer. Furthermore, their results showed a high number of CD34-positive cells in CGFs - CD34 having been demonstrated to play an important role in vascular maintenance, neovascularization and angiogenesis [26].

In theory, CGFs appear to exhibit superior potential for tissue regeneration in clinical and biotechnological applications, as evident in a report of sinus and alveolar ridge augmentation [27]; however, there are few studies supporting this. The purpose of this study was to evaluate the effect of CGFs in the treatment of periodontal intrabony defects.

\section{Materials \& methods Study population}

Seventeen patients (ten females and seven males; aged from 24 to 64 years, average age $47.7 \pm 13.9$ years) suffering from periodontal disease were included in this study. All patients were informed of the nature of this study and signed an informed consent prior to their inclusion. The study was performed in accordance with the Helsinki Declaration of 1975, as revised in 2000, and the study protocol was reviewed and approved by the university ethical board (Peking University, School and Hospital of Stomatology). All patients were treated by the same experienced surgeon at the Department of Periodontology, in the Outpatient Center, at the School and Hospital of Stomatology, Peking University.

Subject inclusion was based on the presence of at least one tooth with a probing depth (PD) of $\geq 6 \mathrm{~mm}$ and radiographic evidence of intrabony defect of $\geq 3 \mathrm{~mm}$ after initial periodontal therapy. Exclusion criteria consisted of patients with systemic diseases, pregnant and/or lactating women, patients taking any drug known to affect the number or function of platelets in the past 3 months and patients with abnormal platelet counts. Teeth nonresponsive and abnormal-responsive to cold stimulation or teeth endodontically treated were also excluded from this study.

\section{Study design}

All patients underwent initial therapy, consisting of oral hygiene instruction, full-mouth scaling and root planning and occlusal adjustment when indicated. After periodontal re-evaluation, the patients were included in this study.

Six patients had a pair of intrabony defects, seven patients had one defect and four patients had three defects (two of the three were a matched pair). In total, 31 defects were included in this study. For the test group, CGFs mixed with BPBM (bovine porous bone mineral, Bio-Oss, Geistilich, Switzerland) was grafted into the intrabony defect; for the control group, BPBM alone was used. Prior to surgery, each defect was randomly assigned to either the test group $(n=15)$ or the control group $(n=16)$ using the randomized block approach. Split-mouth design was used for matched defect pairs; in other words, one defect was assigned according to the random table, and then the matched defect was assigned to the other group. Blood samples were also collected from all patients regardless of CGFs application.

\section{Intra-examiner reproducibility}

Clinical measurements of the included teeth were obtained by the same trained periodontist. Five patients, each showing ten teeth (single- and multirooted) with a PD of $\geq 6 \mathrm{~mm}$ on at least one aspect of each tooth, were used to calibrate the examiner. The examiner evaluated the patients on two separate occasions, $48 \mathrm{~h}$ apart. Intra-observer agreement produced a Cohen's kappa ( $\kappa)$ of 0.859 .

\section{Clinical measurements}

All baseline clinical parameters were obtained on the day of surgery by the same periodontist, who was also 
blind to the type of treatment. Final parameters were taken 1 year postoperatively by the same examiner, again blind to the type of treatment. The following measurements recorded prior to surgery and 1 year after surgery were obtained using the same type of periodontal probe (UNC 15, Hu-Friedy, IL, USA): plaque index [28], bleeding index [29], $\mathrm{PD}$, gingival recession (REC) and clinical attachment level (CAL). The cemento-enamel junction (CEJ) was used as the reference point.

\section{CGF preparation}

CGFs were produced as follow: $9 \mathrm{ml}$ of blood was drawn in sterile Vacuette tubes (Greiner Bio-One, GmbH, Kremsmunster, Austria) without anticoagulant solutions. These tubes were then immediately centrifuged (Medifuge, Silfradentsrl, Sofia, Italy) using a program with the following characteristics: $30 \mathrm{~s}$ acceleration, $2 \mathrm{~min}$ at $2700 \mathrm{rpm}, 4 \mathrm{~min}$ at $2400 \mathrm{rpm}, 4 \mathrm{~min}$ at 2700 $\mathrm{rpm}, 3 \mathrm{~min}$ at $3000 \mathrm{rpm}$ and $36 \mathrm{~s}$ deceleration and stop. At the end of the process, three blood fractions were created (Figure 1A): a superior phase represented by the serum (blood plasma without fibrinogen and coagulation factors, platelet poor plasma, PPP); an interim phase represented by a very large and dense polymerized fibrin block containing the CGFs, white blood cells and stem cells; and the lower RBC layer $[26,30]$.

When used in surgery, the fibrin block and RBC layer beneath it were cut into pieces of $1 \sim 2 \mathrm{~mm}$, and mixed with BPBM granules (particle size $0.25-1.0 \mathrm{~mm}$, BioOss, Geistlich, Wolhusen, Switzerland) at a relative volume of 1:1. This was all mixed and homogenized mechanically for $\sim 6 s$ using the Silfradent Round Up mixer (Silfradent, Italy) to obtain a more uniform mixture. Another part of the fibrin block separated from the red phase was made into a shaped membrane using forceps, and the CGFs membrane was also prepared.

\section{Evaluation of contents of growth factors in CGFs}

Another $9 \mathrm{ml}$ of blood was drawn from the same patient at the same time, and another piece of CGFs was prepared using the method mentioned above. The PPP layer was aspirated and stored at $-20^{\circ} \mathrm{C}$. The fibrin block and RBC layer beneath it were separated and cut into pieces of $1 \sim 2 \mathrm{~mm}$. Then the mixture was centrifuged at $4500 \mathrm{rpm}$ under $4^{\circ} \mathrm{C}$ for $15 \mathrm{~min}$. The supernatant was aspirated and stored at $-20^{\circ} \mathrm{C}$ for measurement of growth factors in it.

The levels of following growth factors in PPP and CGFs were evaluated using double antibody sandwich ELISA: PDGF-BB, TGF- $\beta 1$, IGF-1, VEGF. OD values were measured under $450 \mathrm{~nm}$ wavelength absorbance using microplate reader (Bio-Rad, USA).

\section{Surgical procedure \& intrasurgical measurements}

The same surgeon performed all operations under local anesthesia (4\% articaine with epinephrine 1:100,000). Buccal and lingual sulcular incisions were made, and mucoperiosteal flaps were elevated. Thorough debridement of the defects was achieved with hand instruments. The surgical area was irrigated with copious amounts of sterile saline. During surgery, distance from the CEJ to the most coronal extension of the alveolar bone crest (CEJ-AC) and distance from the $\mathrm{CEJ}$ to the base of the defect (CEJ-BD) were measured using a UNC-15 periodontal probe (Hu-Friedy, USA). Depth of the intrabony defects (AC-BD) was defined as (CEJ-AC)-(CEJ-BD).

During surgery, the prepared mixture of CGFs and BPBM granules was placed into the defects in the CGFs + BPBM group; the mixture was sticky and easy to handle. Following grafting, the CGFs membrane was trimmed and adapted over the entire defect so as to cover 2-3 $\mathrm{mm}$ of the surrounding alveolar bone and to ensure stability of the wound and of the graft material. For control sites, BPBM grafts alone were used. After grafting, the flap was repositioned to accomplish complete interproximal closure. If the closure could not be achieved by the repositioned flap, the coronal reposition flap would be used. The flap was then sutured with 4-0 absorbable sutures with single interrupted sutures and modified vertical mattress, if necessary.

Figure 2 showed No. 4 case in the CGF + BPBM group.

\section{Postoperative care}

All patients were prescribed to take $500 \mathrm{mg}$ amoxicillin three-times a day for 1 week and instructed to rinse with $0.12 \%$ chlorhexidine twice a day for 1 month. Sutures were removed 14 days after the surgery. Patients received professional prophylaxis every 2 weeks during the first 2 months and were followed up with at 3, 4, 5, 6,9 and 12 months after surgery.

\section{Radiographic measurements}

Periapical radiographs were taken with standardized projection geometry at baseline and 1 year after surgery. All radiographs were digitized using a scanner with a transparency module (Hewlett-Packard Scanjet XPA 7400c, Avision, China) to a resolution of 256 pixels with 8 bits of gray-level resolution per pixel and saved as TIFF files. The images were viewed by the same trained radiographer on a $19^{\prime \prime}$ Viewsonic VA703B monitor (Viewsonic, CA, USA) set at a screen resolution of $1280 \times 1024$ pixels. No time restriction was placed on the observer, and images were viewed at 1 -week intervals. The radiographer was blinded to 


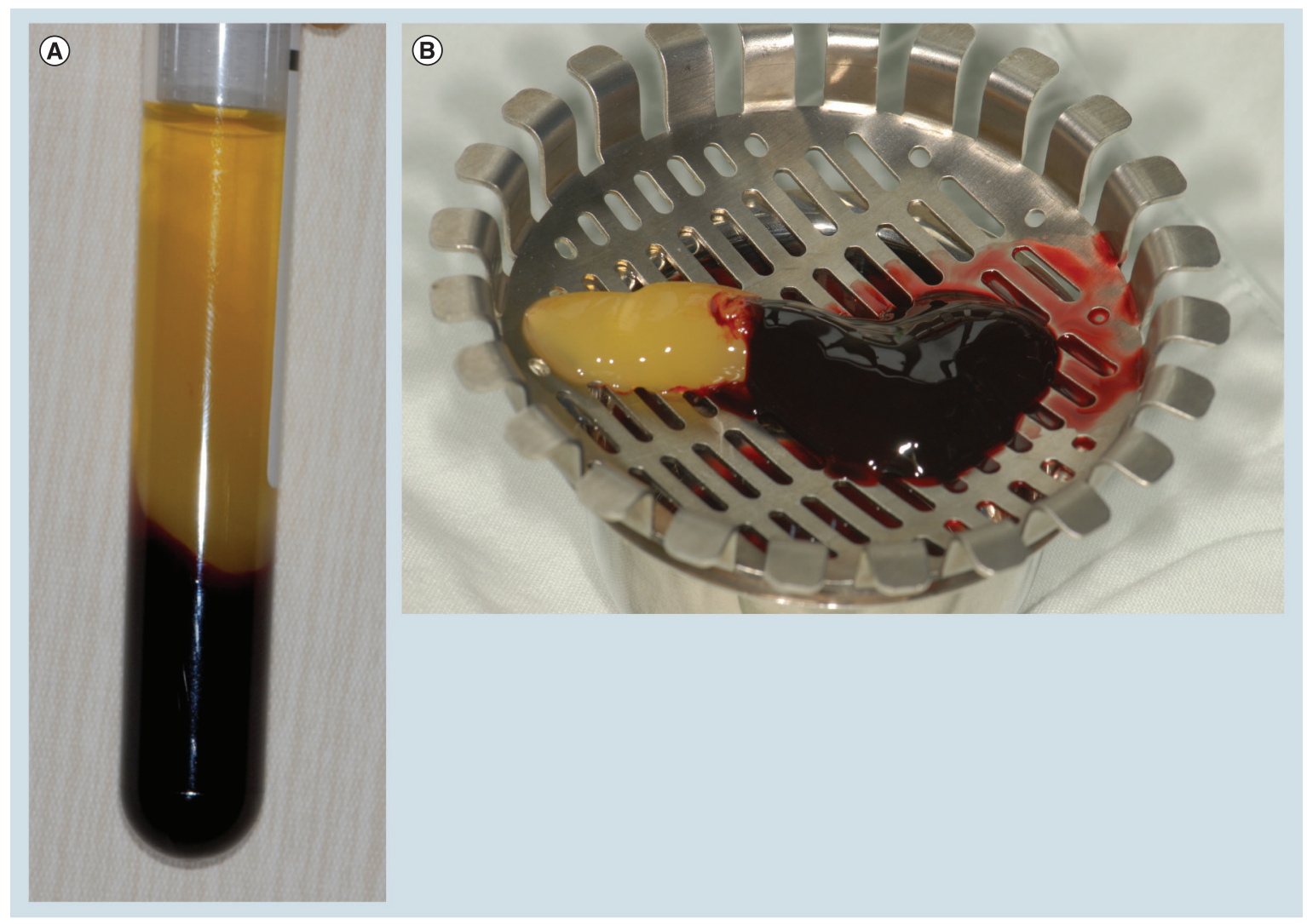

Figure 1. Concentrated growth factors. (A) Three blood fractions were obtained through centrifuge process (1) a superior phase represented by the serum; (2) an interim phase represented by a very large and dense polymerized fibrin block containing the CGFs, white blood cells and stem cells; and (3) the lower red blood cell layer. (B) CGFs separated from platelet-poor plasma.

the treatment. When evaluating the images, the room lights were turned off. Measurements were made to the nearest $0.5 \mathrm{~mm}$ using Photoshop software with a linear measurement tool and a digital magnifying lens. Before the study, the radiographer was calibrated. Intra-observer agreement produced a Cohen's kappa of 0.891 .

The following measurements were performed on both preoperative and postoperative radiographs: distance between the CEJ and alveolar crest (CEJ-AC), distance between the CEJ and base of the defect (CEJ$\mathrm{BD}$ ), and the depth of the intrabony defects - distance between alveolar crest and base of the defect (AC-BD) (Figure 3).

Figure 4 showed the periapical radiographic image of the No. 4 case in the CGF + BPBM group at baseline and 1-year post surgery.

\section{Statistical analysis}

A power analysis determined that a sample size of 30 defects would be sufficient to detect a difference of $5 \%$ or greater between CGFs + BPBM group and $\mathrm{BPBM}$ alone. Results were averaged (mean \pm standard deviation [SD]) for each parameter. SPSS 13.0
(SPSS, IL, USA) was used to analyze the data. Comparisons of baseline clinical and radiographic measurements and of changes from baseline to 1 year between the two treatment groups were performed using paired t-tests. The levels of growth factors in PPP and CGFs were compared using paired t-tests. A p-value of $\leq 0.05$ was considered significant for all statistical analyses.

\section{Results}

All sites healed uneventfully without flap dehiscence or infection. No complications such as abscesses or infections were observed throughout the study period.

\section{Baseline defect comparability}

No differences were observed in the gender distribution between the groups (four females and four males in the CGFs + BPBM group and five females and four males in the BPBM group). The defects displayed a comparable distribution and type in the two groups (Table 1). Intrabony defect depths, as measured during surgery, in the CGFs + BPBM and BPBM groups were not significantly different $(4.6 \pm 1.8 \mathrm{~mm}$ vs $5.0 \pm$ $1.4 \mathrm{~mm}$, respectively) (Table 2). 
Comparison of clinical measurements at baseline \& 1 year post surgery

Plaque index and bleeding index at baseline and 1 year post surgery were not significantly different between the CGFs + BPBM and BPBM groups (Table 3).
Mean PD, REC and CAL at baseline in the CGFs + BPBM and BPBM groups were not significantly different ( $p>0.05)$ (Table 4). Mean PD at baseline was $7.3 \pm 1.5 \mathrm{~mm}$ in the CGFs + BPBM group and $7.5 \pm$ $1.2 \mathrm{~mm}$ in the BPBM group ( $\mathrm{p}>0.05$ ); however, mean

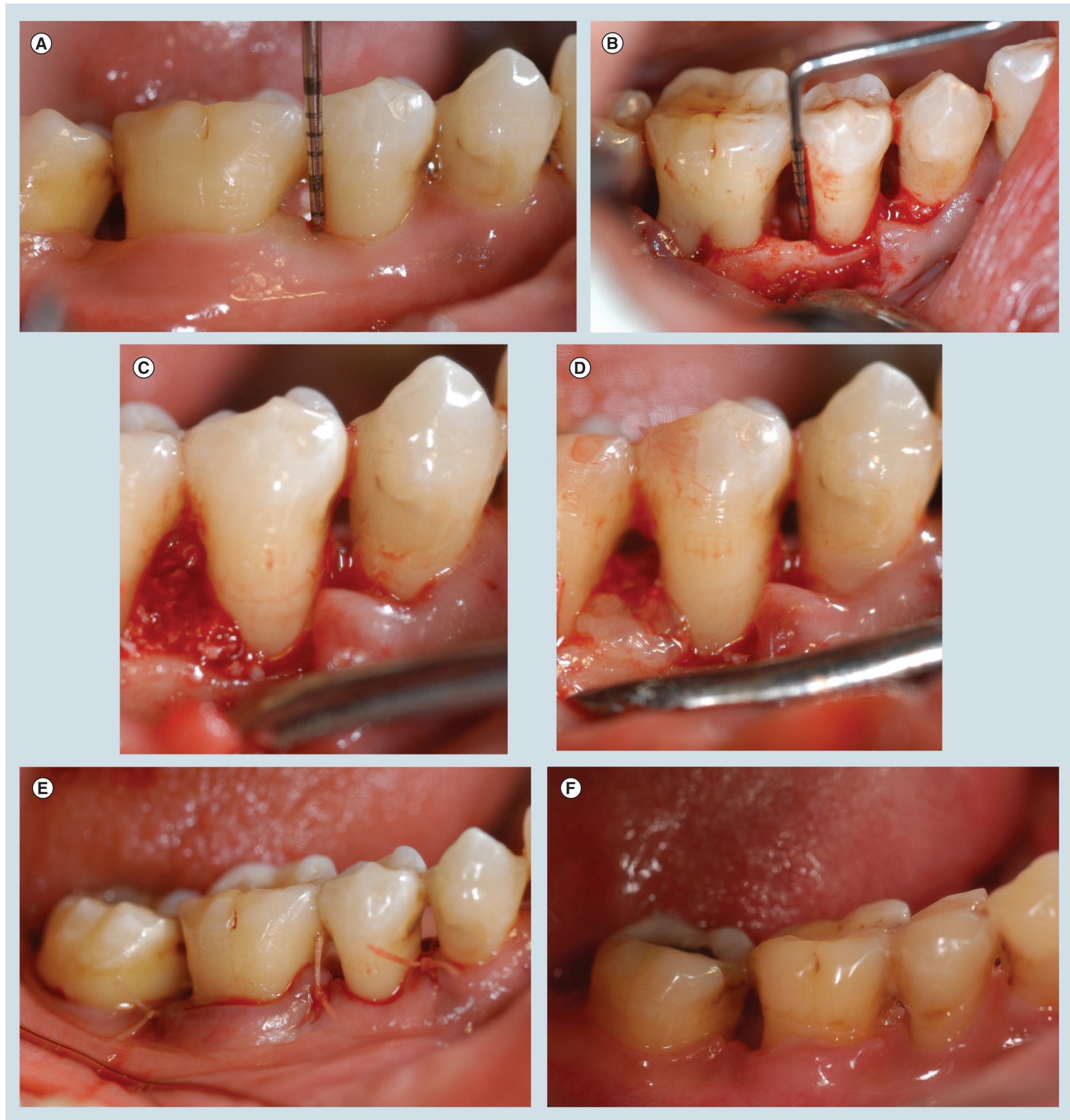

Figure 2. (A) An example in the experimental group: the second right mandibular premolar, baseline. (B) Intra-surgical findings. (C) Mixture of CGFs and BPBM granules was placed into the defect. (D) CGFs membrane was placed. (E) Flap sutured. (F) 1 year post-surgery. 


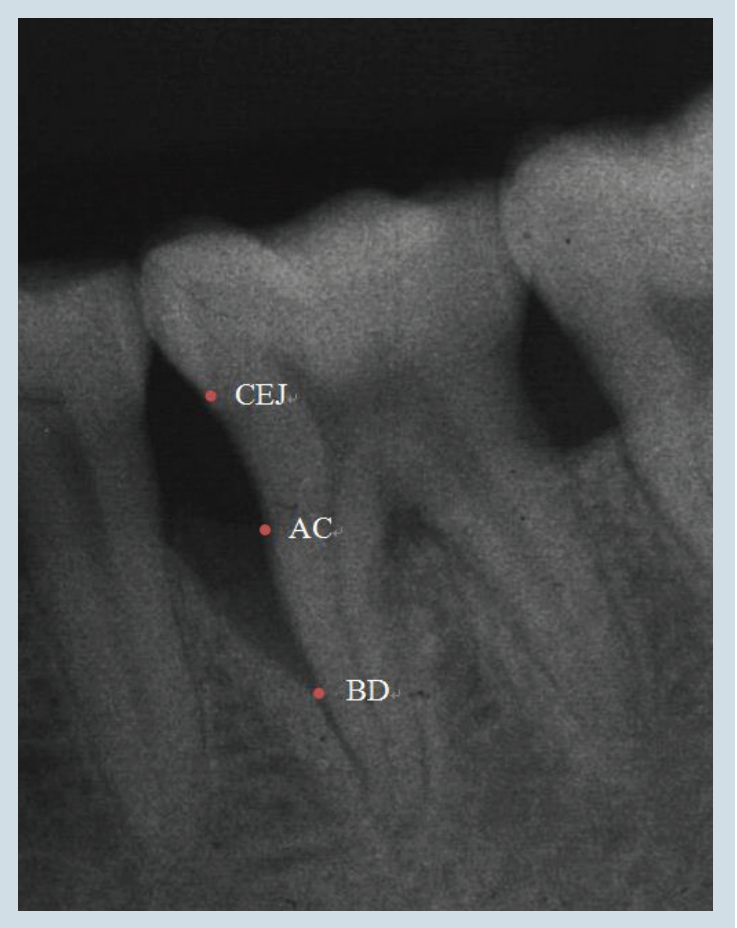

Figure 3. Radiographic landmarks.

AC: Alveolar crest; BD: Base of the defect;

CEJ: Cemento-enamel junction.

PD at 1 year decreased significantly in both groups (decrease of $4.2 \pm 1.3 \mathrm{~mm}$ and $3.0 \pm 1.6 \mathrm{~mm}$, respectively) compared with the baseline data $(\mathrm{p}<0.001)$, and the decrease of mean PD in the CGFs + BPBM group was significantly greater than that of the BPBM group $(\mathrm{p}<0.05)$ (Table 4).

Mean REC at baseline in the CGFs + BPBM and BPBM groups was not significantly different $(p>0.05)$ (Table 4); however, mean REC at1 year increased significantly in both groups (increase of $1.7 \pm 1.5 \mathrm{~mm}$ and $1.8 \pm 1.1 \mathrm{~mm}$, respectively) compared with the baseline data $(p<0.01)$, but neither group exhibited an increase significantly different than the other $(\mathrm{p}>0.05)$.

Mean CAL at baseline in the CGFs + BPBM and BPBM groups was not significantly different $(\mathrm{p}>0.05)$ (Table 4); however, mean CAL at 1 year increased significantly in the CGFs + BPBM group $(3.7 \pm 1.3 \mathrm{~mm})$ $(\mathrm{p}<0.05)$ but not in the BPBM group $(2.4 \pm 1.1 \mathrm{~mm})$ ( $p>0.05$ ). Notably, a $3-\mathrm{mm}$ threshold change was chosen as a cutoff by which to judge a significant clinical improvement. A CAL change of $\geq 3 \mathrm{~mm}$ (significant) occurred in 11 cases $(73.3 \%)$ in the CGFs + BPBM group (Table 5) and in only six cases (37.5\%) in the BPBM group (Table 5).

\section{Comparison of radiographic measurements at} baseline \& 1 year post surgery

There was no statistically significant difference in radiographic measurements between the two groups prior to treatment $(p>0.05)$. Table 6 presents the baseline and 1 year radiographic measurements of the two groups. The mean crestal bone resorption (CEJ-AC)
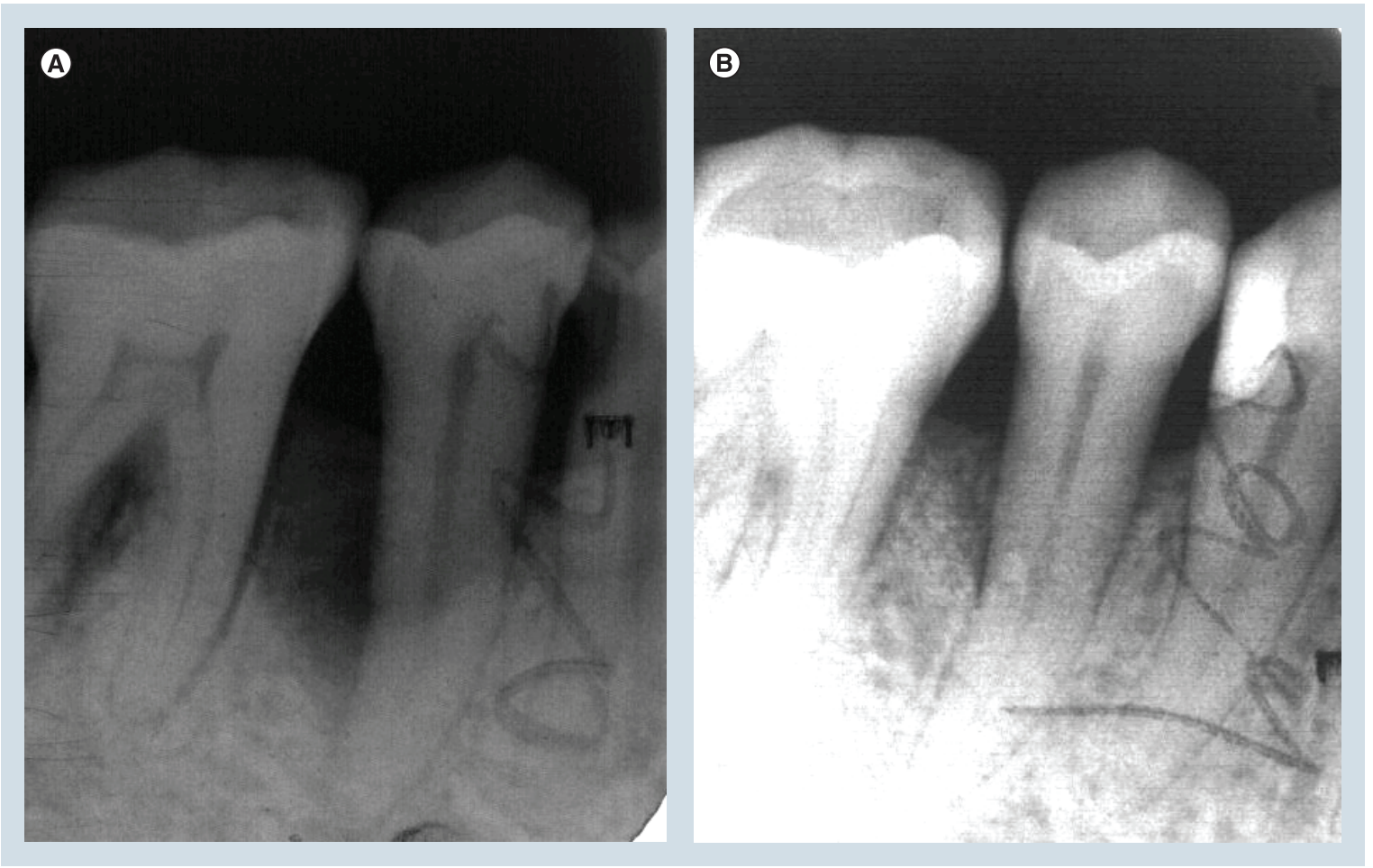

Figure 4. Periapical radiograph of the second right mandibular premolar 1 year post-surgery. 
Table 1. Distribution and type of treated intrabony defects.

\begin{tabular}{|lll|}
\hline Maxilla & CGF + BPBM & BPBM \\
\hline Mandible & 7 & 9 \\
\hline Anterior teeth & 8 & 7 \\
\hline Premolars & 3 & 3 \\
\hline Molars & 3 & 6 \\
\hline 2 wall & 9 & 7 \\
\hline $2-3$ wall & 3 & 4 \\
\hline 3 wall & 6 & 6 \\
\hline BPBM: Bovine porous bone mineral; CGF: Concentrated growth factors. & 6 \\
\hline
\end{tabular}

showed significant differences between baseline and 1 year for both groups $(p<0.001)$. Additionally, significant hard tissue fill (CEJ-BD reduction) occurred in both groups 1 year after treatment $(p<0.001)$. The CGFs + BPBM group presented more favorable gains in radiographic parameters $(3.8 \pm 1.5 \mathrm{~mm})$ than the BPBM group $(2.8 \pm 1.4 \mathrm{~mm})$; however, the difference between the two groups was not significant $(\mathrm{p}>0.05)$.

\section{Comparison of contents of growth factors in CGFs \& PPP}

The average levels of various growth factors in CGFs were significantly higher than that in $\operatorname{PPP}(\mathrm{p}<0.001)$ (Table 7).

\section{Discussion}

This study compared combination CGFs + BPBM with BPBM alone in the treatment of human intrabony defects. The results indicate that CGFs + BPBM treatment was effective in significantly improving clinical and radiographic parameters at 1 year after surgery compared with BPBM alone. However, although significantly different from baseline values, CAL gain and hard tissue fill recorded for the BPBM alone therapy group is limited.

\section{Clinical results}

Few studies exist on the effect of CGFs in the treatment of periodontal intrabony defects. Therefore, a comparison of the data from this study on CGFs + BPBM treatment can only be compared with that of other studies on the use of PRP in the treatment of periodontal intrabony defects.

The improvement in the selected clinical parameters was similar to most previously reported studies. In 2006, we evaluated the effectiveness of PRP as an adjunct to BPBM in the treatment of human intrabony defects and found a statistically significant improvement in the PRP + BPBM group compared with the BPBM group, with a PD reduction of $4.78 \mathrm{~mm}$ and a CAL gain of $4.52 \mathrm{~mm}$ [31]. Lekovic et al. compared the clinical effectiveness of two regenerative techniques $(P R P+G T R+B P B M$ vs PRP + BPBM) for intrabony defects in humans [16]. At 6 months post surgery, both treatments showed significant improvement compared with the baseline values with no statistically significant difference. For the PRP + BPBM group, PD reduction was $3.98 \mathrm{~mm}$ on buccal sites and $3.94 \mathrm{~mm}$ on lingual sites, and CAL gain was $3.78 \mathrm{~mm}$ on buccal sites and $3.84 \mathrm{~mm}$ on lingual sites. In a 6 month clinical trial, Hanna et al. compared the clinical outcomes obtained by PRP + BPBM to those obtained from BPBM alone in the treatment of periodontal intrabony defects. They reported $3.54 \mathrm{~mm}$ of $\mathrm{PD}$ reduction and $3.15 \mathrm{~mm}$ of CAL gain in the PRP + BPBM group, each significantly greater than that of the BPBM group [17]. Parimala $e t a l$. compared the 9 months efficacy of BPBM with and without PRP for the treatment of 28 human periodontal intrabony defects, noting a PD reduction of $6.60 \mathrm{~mm}$ for the PRP + BPBM-treated sites and a CAL gain of $4.70 \mathrm{~mm}$ for the PRP + BPBM group [32]; their results appeared to be more favorable than those of other studies.

Table 2. Mean intrabony defect characteristics in surgery.

\begin{tabular}{|lccl|}
\hline Treatment & CEJ-BD $(\mathrm{mm})$ & CEJ-AC $(\mathrm{mm})$ & AC-BD $(\mathrm{mm})$ \\
CGF + BPBM $(\mathrm{n}=15)$ & $9.1 \pm 1.1$ & $4.5 \pm 1.6$ & $4.6 \pm 1.8$ \\
\hline BPBM $(\mathrm{n}=16)$ & $8.6 \pm 1.5$ & $3.6 \pm 1.4$ & $5.0 \pm 1.4$ \\
\hline AC: Alveolar bone crest; AC-BD: Depth of intrabony defect; BD: Base of defect; CEJ: Cemento-enamel junction.
\end{tabular}




\begin{tabular}{|c|c|c|}
\hline & CGFs + BPBM & BPBM \\
\hline \multicolumn{3}{|l|}{ Plaque index } \\
\hline Baseline & $0.7 \pm 0.3$ & $0.8 \pm 0.4$ \\
\hline 1 year post surgery & $0.7 \pm 0.2$ & $0.8 \pm 0.5$ \\
\hline \multicolumn{3}{|l|}{ Bleeding index } \\
\hline Baseline & $0.9 \pm 0.6$ & $0.9 \pm 0.5$ \\
\hline 1 year post surgery & $1.0 \pm 0.7$ & $1.0 \pm 0.7$ \\
\hline
\end{tabular}

A $3 \mathrm{~mm}$ threshold change was chosen as a cutoff by which to judge a significant clinical improvement. A CAL change of $\geq 3 \mathrm{~mm}$ occurred in $73.3 \%$ of patients in the CGFs + BPBM group in the present study, while Hanna et al. previously noted CAL changes of $\geq 3 \mathrm{~mm}$ $76.9 \%$ (ten cases) of patients in their PRP + BPBM group.

\section{Hard tissue fill}

In this study, the average radiographic hard tissue fill is in accordance with previous reports on the clinical benefits of a regenerative approach employing PRP combined with BPBM.

Parimala et al. reported a significant radiographic defect hard tissue fill of $4.04 \mathrm{~mm}$ in the PRP + BPBM group [32]. In our 2006 study, bone probing level (BPL), measured with a UNC-15 probe under local anesthesia, was used to determine hard tissue fill; BPL reduction was $4.56 \mathrm{~mm}$ in the PRP + BPBM group [31]. See comment in PubMed Commons below, Lekovic et al. evaluated defect hard tissue fill by re-entry surgery at 6 months after regenerative surgery and revealed a reduction of $4.82 \mathrm{~mm}$ on buccal sites and of $4.74 \mathrm{~mm}$ on lingual sites in the PRP + BPBM group [16]. Notably, each study characterized intrabony defects differently and evaluated bone level using different methods (radiography, bone probing and re-entry surgery). Projection geometry may have also been different. Therefore, a more accurate, standardized method to evaluate defect hard tissue fill in regenerative treatment is needed.

\section{Possible CGFs mechanism of action}

In the present study, inclusion of CGFs improved the outcome of regenerative periodontal intrabony defect treatment. CGFs activity in this respect may be due to its biologic constituents, in addition to chemotactic and mitogenic PDGF, that are involved in tissue regeneration.

\begin{tabular}{|c|c|c|c|}
\hline & CGF + BPBM $(n=15)$ & BPBM $(n=16)$ & $p$-value \\
\hline \multicolumn{4}{|l|}{ Probing depth (mm) } \\
\hline Baseline & $7.3 \pm 1.5$ & $7.5 \pm 1.2$ & 0.617 \\
\hline 1 year post surgery & $3.1 \pm 0.7$ & $4.5 \pm 0.8$ & 0.001 \\
\hline Difference & $4.2 \pm 1.3$ & $3.0 \pm 1.6$ & 0.016 \\
\hline$p$-value & $<0.001$ & $<0.001$ & \\
\hline \multicolumn{4}{|c|}{ Gingival recession $(\mathrm{mm})$} \\
\hline Baseline & $1.3 \pm 1.2$ & $1.2 \pm 0.9$ & 1.000 \\
\hline 1 year post surgery & $1.7 \pm 1.5$ & $1.8 \pm 1.1$ & 0.847 \\
\hline Difference & $0.5 \pm 0.6$ & $0.7 \pm 0.5$ & 0.595 \\
\hline$p$-value & 0.006 & $<0.001$ & \\
\hline \multicolumn{4}{|c|}{ Clinical attachment level (mm) } \\
\hline Baseline & $8.5 \pm 2.3$ & $8.7 \pm 1.9$ & 0.767 \\
\hline 1 year post surgery & $4.8 \pm 2.0$ & $6.3 \pm 1.6$ & 0.041 \\
\hline Difference & $3.7 \pm 1.3$ & $2.4 \pm 1.1$ & 0.013 \\
\hline$p$-value & $<0.001$ & $<0.001$ & \\
\hline
\end{tabular}


Table 5. Number of sites and percentage of clinical attachment level gain $\geq 3 \mathrm{~mm}$ in test and control groups.

\begin{tabular}{|lll|}
\hline CAL gain $\geq \mathbf{3} \mathbf{~ m m}$ & CGF + BPBM $(\mathbf{n}=\mathbf{1 5})$ & BPBM $(\mathbf{n}=\mathbf{1 6})$ \\
Sites & 11 & 6 \\
\hline Percentage & $73.3 \%$ & $37.5 \%$ \\
\hline BPBM: Bovine porous bone mineral; CAL: Clinical attachment level; CGF: Concentrated growth factor. \\
\hline
\end{tabular}

Use of different centrifugation speeds permits the collection of abundant growth factors located just below the buffy coat and above the dense clot portion. The results of the present study showed that the levels of TGF- $\beta 1$, PDGF-BB, IGF-1 and VEGF in CGFs were significantly higher than those in PPP $(p<0.001)$. Rodella et al. confirmed the presence of TGF- $\beta 1$ and VEGF in CGFs and demonstrated a similar pattern of expression in the RBC layer [26]. Polypeptide growth factors have been shown to play an important role in the growth and differentiation of cells involved in periodontal wound healing $[10,11,33]$. Unlike PRP, CGFs do not dissolve rapidly following application. Instead, the strong fibrin gel in the matrix addition is slowly remodeled in a similar manner to a natural blood clot. Thus, CGFs prolong the duration of growth factor activity, which is conducive for growth factor synergy, and enhances cell proliferation and osteogenic differentiation [30].

Another likely favorable component in CGFs are stem cells. Using immunohistochemistry, Rodella et al. found a higher number of CD34-positive cells in CGF layers than in RBC layers, possibly trapped by the CGF network composition [26]. PRF are known to provide a supportive matrix for circulating mesenchymal stem cells, which are recruited from blood to injured tissue by signaling molecules released from platelets [34]. Moreover, increasing evidence points to the role of circulating CD34-positive cells in vascular maintenance, neovascularization and angiogenesis [35-37].

The fibrin buffy coat is a major component in CGFs. In the present study, the fibrin block is cut into pieces of approximately $1-2 \mathrm{~mm}$, mixed with BPBM and put into the bone defects. It is well known that in periodontal regeneration, unimpeded absorption, adhesion and maturation of the fibrin clot for formation of a connective tissue attachment over a long junctional epithelium is vitally important [38]. The CGFs not only improve the wound stability, which is essential for the establishment of a new connective tissue attachment to a root surface, but also provide a scaffold supporting cytokine attachment and cellular migration. Through

Table 6. Mean radiographic measurements of test and control groups.

\begin{tabular}{|c|c|c|c|}
\hline & CGFs + BPBM $(n=15)$ & BPBM $(n=16)$ & p-value \\
\hline \multicolumn{4}{|l|}{ CEJ-AC (mm) } \\
\hline Baseline & $4.5 \pm 1.4$ & $3.6 \pm 1.5$ & 0.245 \\
\hline 1 year post surgery & $4.9 \pm 1.6$ & $4.4 \pm 1.5$ & 0.421 \\
\hline Difference & $0.5 \pm 0.3$ & $0.8 \pm 0.5$ & 0.222 \\
\hline$p$-value & $<0.001$ & $<0.001$ & \\
\hline \multicolumn{4}{|l|}{ CEJ-BD (mm) } \\
\hline Baseline & $9.3 \pm 1.1$ & $8.4 \pm 1.9$ & 0.234 \\
\hline 1 year post surgery & $6.1 \pm 1.3$ & $6.4 \pm 1.6$ & 0.580 \\
\hline Difference & $3.3 \pm 1.5$ & $2.1 \pm 1.5$ & 0.087 \\
\hline$p$-value & $<0.001$ & $<0.001$ & \\
\hline \multicolumn{4}{|l|}{ AC-BD (mm) } \\
\hline Baseline & $4.9 \pm 1.8$ & $4.8 \pm 1.8$ & 0.935 \\
\hline 1 year post surgery & $1.1 \pm 1.6$ & $1.9 \pm 0.6$ & 0.002 \\
\hline Difference & $3.8 \pm 1.5$ & $2.8 \pm 1.4$ & 0.153 \\
\hline$p$-value & $<0.001$ & $<0.001$ & \\
\hline
\end{tabular}


Table 7. The levels of growth factors in platelet poor plasma and concentrated growth factors.

\begin{tabular}{|llll|}
\hline & PPP & CGFs & p-value \\
\hline PDGF-BB $(\mathrm{ng} / \mathrm{ml})$ & $37.58 \pm 12.12$ & $176.88 \pm 52.32$ & $<0.001$ \\
\hline TGF- $\beta 1(\mathrm{ng} / \mathrm{ml})$ & $65.34 \pm 21.21$ & $703.02 \pm 86.77$ & $<0.001$ \\
\hline IGF-1 $(\mathrm{ng} / \mathrm{ml})$ & $73.02 \pm 18.37$ & $533.69 \pm 67.35$ & $<0.001$ \\
\hline VEGF $(\mathrm{pg} / \mathrm{ml})$ & $44.77 \pm 10.05$ & $231.36 \pm 44.01$ & $<0.001$ \\
\hline CGF: Concentrated growth factor; PPP: Platelet poor plasma. & & \\
\hline
\end{tabular}

the polymerization of the fibrinogen molecules, the fibrin block comprises a 3D polymer network of interwoven fibers. Upon scanning electron microscopic analysis of the fibrin block, Rodella et al. observed a fibrin network constituted by thin and thick fibrillar elements, including multiple trapped platelets. A 3D environment is crucial for cell-cell and protein-protein interactions to create tissue symmetry [26].

To date, there is only one published study regarding the effect of CGFs on periodontal ligament stem cells (PDLSCs) in vitro. Yu B et al. investigated the proliferation and differentiation of beagle PDLSCs cocultured with CGFs and showed that CGFs significantly promoted the proliferation of PDLSCs and exhibited a dose-dependent effect on the activation and differentiation of the stem cells [30]. These data appear to support the hypothesis in the current study; however, future studies are needed to better characterize the mechanism underlying CGFs activity and to support its clinical application.

\section{The limitation of the present study}

There were 31 defects in 17 patients included in this study. Seven patients had only one intrabony defect, which cannot be used in a split-mouth design. A larger sample size and a more rigorous overall experimental design are necessary for future studies. The methods we used to evaluate the regenerative effect included clinical examination and periapical radiographs. Although a good intra-examiner reproducibility was obtained, the re-entry measurement is still the 'gold standard' to reflect actual bone change; and periapical radiography is inherently limited in its ability to evaluate true bone level. Furthermore, the suture technique of the surgeon could also affect the outcome of the treatment.

\section{Conclusion}

In conclusion, the results of this study indicate that the CGFs + BPBM combination improved clinical outcome better than BPBM alone in the treatment of human intrabony defects. This also appears the case for hard tissue fill, CAL gain and PD reduction. The CGFs + BPBM group presented more favorable, but not statistically significant, gains in radiographic hard tissue fill. Long-term, multicenter randomized, controlled clinical trials will be required to better understand the clinical and radiographic effects of CGFs on periodontal regeneration.

\section{Author contribution}

J Qiao and C Sun designed the present study. C Sun completed all the surgeries. J Qiao was responsible for the clinical examination. C Sun completed all the surgeries. J Duan was responsible for the radiographic measurements. Y Chu completed the data records and statistical analysis. J Qiao and $Y$ Zhang completed the paper writing work.

\section{Acknowledgements}

The authors would like to thank the members of the Department of Periodontology and Radiology for their help in the current investigation. The authors also acknowledge the help from M Zhang and S Wang.

Financial \& competing interests disclosure

J Qiao accepted the support by Ministry of Science and Technology of China under contract International Science \& Technology Cooperation Program foundation Nr.1019. The authors have no other relevant affiliations or financial involvement with any organization or entity with a financial interest in or financial conflict with the subject matter or materials discussed in the manuscript apart from those disclosed.

No writing assistance was utilized in the production of this manuscript.

\section{Ethical conduct of research}

The authors state that they have obtained appropriate institutional review board approval or have followed the principles outlined in the Declaration of Helsinki for all human or animal experimental investigations. In addition, for investigations involving human subjects, informed consent has been obtained from the participants involved.

\section{Open access}

This work is licensed under the Creative Commons Attribution 4.0 License. To view a copy of this license, visit http:// creativecommons.org/licenses/by/4.0/ 
Executive summary

- Addition of concentrated growth factors significantly improved the clinical effectiveness of bovine porous bone mineral to treat intrabony defects.

- Long-term, multicenter randomized, controlled clinical trials will be required.

\section{References}

Papers of special note have been highlighted as: $\bullet$ of interest; •• of considerable interest

1 Cortellini P, Tonetti MS. Long-term tooth survival following regenerative treatment of intrabony defects. J. Periodontol. 75(5), 672-678 (2004).

2 Sculean A, Pietruska M, Schwarz F et al. Healing of human intrabony defects following regenerative periodontal therapy with an enamel matrix protein derivative alone or combined with a bioactive glass. A controlled clinical study. J. Clin. Periodontol. 32(1), 111-117 (2005).

3 Artzi Z, Tal H, Platner O et al. Deproteinized bovine bone in association with guided tissue regeneration or enamel matrix derivatives procedures in aggressive periodontitis patients: a 1 year retrospective study. J. Clin. Periodontol. 42(6), 547-456 (2015).

4 Tonetti MS, Cortellini P, Lang NP et al. Clinical outcomes following treatment of human intrabony defects with GTR/ bone replacement material or access flap alone. A multicenter randomized controlled clinical trial. J. Clin. Periodontol. 31(9), 770-776 (2004).

5 Sanz M, Tonetti MS, Zabalegui I et al. Treatment of intrabony defects with enamel matrix proteins or barrier membranes: results from a multicenter practice-based clinical trial. J. Periodontol. 75(5), 726-733 (2004).

6 MacNeil RL, Somerman MJ. Development and regeneration of the periodontium: parallels and contrasts. Periodontol. 2000 19, 8-20 (1999).

7 Kornman KS, Robertson PB. Fundamental principles affecting the outcomes of therapy for osseous lesions. Periodontol. 2000 22, 22-43 (2000).

8 Sculean A, Windisch P, Keglevich T et al. Clinical and histologic evaluation of humanintrabony defects treated with an enamel matrix proteinderivative combined with a bovinederived xenograft. Int. J. Periodontics Restorative Dent. 23(1), 47-55 (2003)

9 Susin C, Fiorini T, Lee J et al. Wound healing following surgical and regenerative periodontal therapy. Periodontol. 2000 68, 83-98 (2015).

10 Lynch SE, Williams RC, Polson AM et al. A combination of platelet-derived and insulin-like growth factors enhances periodontal regeneration. J. Clin. Periodontol. 16(8), 545-548 (1989).

11 Giannobile WV, Finkelman RD, Lynch SE. Comparison of canine and non-human primate animal models for periodontal regenerative therapy: results following a single administration of PDGF/IGF-I. J. Periodontol. 65(12), 1158-1168 (1994).

12 Borzini P, Mazzucco L. Platelet gels and releasates. Curr. Opin. Hematol. 12(6), 473-479 (2005).
13 Whitman DH, Berry RL, Green DM. Platelet gel: anautologous alternative to fibrin glue with applications in oral and maxillofacial surgery. J. Oral Maxillofac. Surg. 55(11), 1294-1299 (1997).

- This was the first time that PRP was introduced into oral surgery.

14 Marx RE, Carlson ER, Eichstaedt RM et al. Platelet-rich plasma: growth factor enhancement for bone grafts. Oral Surg. Oral Med. Oral Pathol. Oral Radiol. Endod. 85(6), 638-646 (1998).

- This was the first time that PRP was introduced into periodontal region.

15 de Obarrio JJ, Araúz-Dutari JI, Chamberlain TM et al. The use of autologous growth factors in periodontalsurgical therapy: platelet gel biotechnology - case reports. Int. J. Periodontics Restorative. Dent. 20(5), 486-497 (2000).

16 Lekovic V, Camargo PM, Weinlaender M et al. Comparison of platelet-rich plasma, bovine porous bone mineral, and guided tissue regeneration versus platelet-rich plasma and bovine porous bone mineral in the treatment of intrabony defects: a reentry study. J. Periodontol. 73(2), 198-205 (2002).

17 Hanna R, Trejo PM, Weltman RL. Treatment of intrabony defects with bovine-derived xenograft alone and in combination with platelet-rich plasma: a randomized clinical trial. J. Periodontol. 75(12), 1668-1677 (2004).

18 Camargo PM, Lekovic V, Weinlaender M et al. A reentry study on the use of bovine porous bone mineral, GTR, and platelet-rich plasma in the regenerative treatment of intrabony defects in humans. Int. J. Periodontics Restorative. Dent. 25(1), 49-59 (2005)

19 Döri F, Arweiler N, Húszár T et al. Five-year results evaluating the effects of platelet-rich plasma on the healing of intrabony defects treated with enamel matrix derivative and natural bone mineral. J. Periodontol. 84(11), 1546-1555 (2013).

20 Pinipe J, Mandalapu NB, Manchala SR et al. Comparative evaluation of clinical efficacy of $\beta$-tri calcium phosphate (Septodont-RTR) ${ }^{\mathrm{TM}}$ alone and in combination with platelet rich plasma for treatment of intrabony defects in chronic periodontitis. J. Indian Soc. Periodontol. 18(3), 346-351 (2014).

21 Dohan DM, Choukroun J, Diss A et al. Platelet-rich fibrin (PRF): a second-generation platelet concentrate. Part I: technological concepts and evolution. Oral Surg. Oral Med. Oral Pathol. Oral Radiol. Endod. 101(3), e37-e44 (2006).

- Classical literatures on platelet-rich fibrin (PRF).

22 Dohan DM, Choukroun J, Diss A et al. Platelet-rich fibrin (PRF): a second-generation platelet concentrate. Part II: platelet-related biologic features. Oral Surg. Oral Med. Oral Pathol. Oral Radiol. Endod. 101(3), e45-e50 (2006).

- Classical literatures on PRF. 
23 Dohan DM, Choukroun J, Diss A et al. Platelet-rich fibrin (PRF): a second-generation platelet concentrate. Part III: leucocyte activation: a new feature for platelet concentrates? Oral Surg. Oral Med. Oral Pathol. Oral Radiol. Endod. 101, e51-e55 (2006).

- Classical literatures on PRF.

24 Choukroun J, Diss A, Simonpieri A et al. Platelet-rich fibrin (PRF): a second-generation platelet concentrate. Part IV: clinical effects on tissue healing. Oral Surg. Oral Med. Oral Pathol. Oral Radiol. Endod. 101(3), e56-e60 (2006).

- Classical literatures on PRF.

25 Choukroun J, Diss A, Simonpieri A et al. Platelet-rich fibrin (PRF): a second-generation platelet concentrate. Part V: histologic evaluations of PRF effects on bone allograft maturation in sinus lift. Oral Surg. Oral Med. Oral Pathol. Oral Radiol. Endod. 101(3), 299-303 (2006).

- Classical literatures on PRF.

26 Rodella LF, Favero G, Boninsegna R et al. Growth factors, CD34 positive cells, and fibrin network analysis in concentrated growth factors fraction. Microsc. Res. Tech. 74(8), 772-777 (2011).

-• This was the first time to report CGFs.

27 Sohn DS, Heo JU, Kwak DH et al. Bone regeneration in the maxillary sinus using an autologous fibrin-rich block with concentrated growth factors alone. Implant Dent. 20(5), 389-395 (2011).

28 Löe $\mathrm{H}$. The gingival index, the plaque index and the retention index systems. J. Periodontol. 38(6), 610-616 (1967).

29 Mazza JE, Newman MG, Sims TN. Clinical and antimicrobial effect of stannous fluoride on periodontitis. J. Clin. Periodontol. 8(3), 203-212 (1981).
30 Yu B, Wang Z. Effect of concentrated growth factors on beagle periodontal ligament stem cells in vitro. Mol. Med. Rep. 9(1), 235-242 (2014).

-• This was the first time to investigate the role of CGFs on periodontal ligament stem cells.

31 Parimala M, Mehta DS. Comparative evaluation of bovine porous bone mineral. J. Indian Soc. Periodontol. 14(2), 126-131 (2010).

32 Howell TH, Fiorellini JP, Paquette DW et al. A Phase I/ II clinical trial to evaluate a combination of recombinant human platelet-derived growth factor-BB and recombinant human insulin-like growth factor-I in patients with periodontal disease. J. Periodontol. 68(12), 1186-1193 (1997).

33 Gassling VL, Açil Y, Springer IN et al. Platelet-rich plasma and platelet-rich fibrin in human cell culture. Oral Surg. Oral Med. Oral Pathol. Oral Radiol. Endod. 108(1), 48-55 (2009).

34 Matic M, Petrov IN, Chen S et al. Stem cells of the corneal epithelium lack connexins and metabolite transfer capacity. Differentiation 61(4), 251-260 (1997).

35 Ademokun JA, Chapman C, Dunn J et al. Umbilical cord blood collection and separation for haematopoietic progenitor cell banking. Bone Marrow Transplant. 19(10), 1023-1028 (1997).

36 Kikuchi-Taura A, Soma T, Matsuyama T et al. A new protocol for quantifying CD34(+) cells in peripheral blood of patients with cardiovascular disease. Tex. Heart Inst. J. 33(4), 427-429 (2006)

37 Hiatt WH, Stallard RE, Butler ED et al. Repair following mucoperiosteal flap surgery with full gingival retention. J. Periodontol. 39(1), 11-16 (1968).

38 Polson AM, Proye MP. Fibrin linkage: a precursor for new attachment. J. Periodontol. 54(3), 141-147 (1983). 